\title{
Novel Technologies to Study Smoking Behavior: Current Developments in Ecological Momentary Assessment
}

\author{
Natalie Schüz • John Cianchi • Saul Shiffman • \\ Stuart G. Ferguson
}

Published online: 18 January 2015

(C) Springer International Publishing AG 2015

\begin{abstract}
Ecological momentary assessment (EMA) methods for the capture of real-world data in real time have seen significant advances, both in popularity and application, over the past few years. As a research methodology, EMA offers considerable advantages and has proven to be particularly suited to the study of substance dependence research. Using EMA, research participants can record information about events, symptoms, situations, thoughts, feelings, and behaviors as they occur. Moreover, when used in concert with other technologies, such as geospatial software, EMA becomes a tool with which researchers can gather rich data about individuals' daily lives. This review aims to introduce the reader to EMA and to the rationale behind its use. We discuss the applications for which EMA can be used, and the many advantages they offer, with a particular focus on the study of smoking behaviors.
\end{abstract}

Keyword Ecological momentary assessment · Smoking · Dependence - Addiction · Real-time data capture ·

Research methodology $\cdot$ Technology

This article is part of the Topical Collection on Tobacco

N. Schüz $(\square) \cdot J$. Cianchi $\cdot$ S. G. Ferguson

School of Medicine, University of Tasmania, Private Bag 34,

Hobart, Tasmania 7001, Australia

e-mail: natalie.schuez@utas.edu.au

S. Shiffman

University of Pittsburgh, Pittsburgh, PA, USA

\section{Introduction}

Assessment of smoking behavior often requires detailed knowledge of an individual's patterns of smoking, including the relationship between smoking and situational factors. Ecological momentary assessment (EMA) often uses modern technology to accomplish this goal. EMA refers to the repeated collection of data in the daily life of study participants. Individuals record momentary or "real-time", information about events, symptoms, situations, thoughts, feelings, and behaviors within the environments in which they naturally occur $[1,2]$; this provides researchers with self-reported data that not only overcome some of the recall problems that plague data that is recorded retrospectively, but also enable exploration of the role of environmental context in influencing a person's experience and behavior. Furthermore, EMA enables participants to record events as they unfold over time, providing researchers with insight into the temporal resolution of dynamic processes that may be unavailable using alternative methods [3].

EMA methods have been extensively and successfully applied to the study of smoking behavior. A search of published studies found 239 EMA articles relating to smoking in the last decade alone, 123 of which were published in the last 3 years. These studies have helped to elucidate the role of social and situational factors in maintaining smoking and as precipitating factors in smoking cessation failure. EMA studies have allowed researchers to study the process of quitting smoking in detail, leading to both a better understanding of how and why attempts to quit fail, and to the improvement of quitting methods and techniques.

The purpose of this paper is to review recent advances in the study of health behaviors to provide an introduction to EMA with a particular emphasis on its utility as a research methodology for the study of smoking behaviors. In addition, it briefly reviews the various technologies associated with 
EMA-based research as well as the considerations researchers must make when designing EMA protocols.

\section{Background}

The term EMA was coined in 1994 [1], however, research that would now be recognized as EMA has been undertaken for considerably longer within a range of social research disciplines. For example, up until the 1980s, paper-and-pencil diaries were used to record all manner of phenomena, including daily reports of disease symptoms, medication intake, health behaviors, or general well being. A major change that has occurred within the last two decades, however, has been the increased availability of handheld electronic technologies (i.e., PDAs, smartphones, tablets) that are now commonly used in EMA research to collect data and monitor participants. This advance in technology, and the development of conceptual frameworks and analytic methods for such data, significantly advanced the popularity and utility of EMA methods.

Research methods that are today termed "EMA" share a number of common features. Specifically, assessments are completed in real time (momentary), on multiple occasions (repetitive), and within the environment in which they naturally occur [2]. Typically, EMA designs sample "moments" throughout participants' day-to-day lives, often on the basis of random or regularly occurring schedules, by way of surveys or diary entries administered via handheld computers or smartphones [2]. In addition, EMA is also used, often concurrently, to sample targeted events of interest, such as the smoking of a cigarette [2]. While EMA studies may differ wildly in terms of design, all are momentary, repetitive, and completed within the participant's natural environment.

\section{Advantages of EMA}

EMA methods grew from the need for an assessment tool that could overcome the failings of many existing data collection techniques, such as recall biases in retrospective studies, and the artificial nature of laboratory studies. Thus, EMA offers a number of advantages over alternate methodologies. Firstly, assessments are "ecological" because data are recorded in the participants' natural environments, rather than in a laboratory setting where social and environmental contexts that affect people's experiences and behaviors are absent. Indeed, many behavioral events simply cannot be simulated in a laboratory setting [e.g., 4]. Consequently, researchers may be confident that their findings reflect what is happening in participants' everyday life [2]. For example, in a study examining self-control during craving episodes among smokers, Berkman, Falk, and Lieberman [5•] used EMA in conjunction with functional neuroimaging and were able to show that neurocognitive data collected in the laboratory relate to real-world instances of self-control. This study not only shows a way of linking behavioral observations with neuroscience research but also provides important evidence for the ecological validity of brain imaging data.

EMA methods are also "momentary;" data pertaining to variables of interest is collected in the moment in which it is experienced, rather than being recorded at a later time. These momentary assessments are also completed multiple times within a period, allowing the researcher to track behaviors or experiences across time and contexts. The key concept underlying EMA is that it can sample dynamic processes as they proceed through their "life cycle," providing insight into the antecedents and consequences of events, behaviors, and experiences.

Perhaps the most important benefit of momentary assessment is that, by collecting data when the target event occurs, problems with memory recall and bias are minimized $[2-4,5 \bullet$, 6]. Retrospective reports, completed even a few minutes after target events, may be inaccurate and prone to biases that are influenced by personal history, context, and the person's mental state when giving their report [e.g., 7-9]. EMA provides insights into experience in the moment, whereas retrospective self-reports provide insights into how the individual constructs, or reconstructs, inevitably biased memories and meanings about experiences, see also [6, 7]. EMA is, therefore, particularly suited to learning about individuals as they respond to their environment moment-by-moment [7].

In summary, EMA offers three main advantages. Firstly, it enables the researcher to study within-individual processes as they unfold in the participants' normal environment, yielding ecologically valid data. Further, repeated measures provide a greater understanding of the natural history of the target process(es) than would otherwise be available, and the resulting data allows for both descriptive and causal analyses. Finally, data are recorded in near-real time, overcoming the disadvantages of retrospective methods, particularly memory bias.

\section{Applications}

EMA has been used to study a variety of research questions across a number of disciplines. The common factor being that they involve the assessment of within-individual dynamic processes, such as the frequent, within-daily variations in measurable phenomena that often occur in the study of behaviors (e.g., smoking cessation). The following section will review some of the major applications of EMA in health and social behavioral research, with specific attention to their utility in the study of smoking behaviors.

\section{Temporal Sequences}

EMA has great utility as a means of exploring the temporal sequences behind events; that is, the manner in which events 
unfold over time and context [for different conceptualizations of time within the EMA framework, see 10]. Moreover, EMA can uncover the dynamic relationships that exist between variables and their antecedents, which are difficult to detect if measurements are made only a few times. This is particularly valuable for the development of improved treatment options. In a prospective study exploring the temporal effects of stress and negative effect on smoking lapses, Shiffman and Waters [11] collected real-time data on over 100 lapse episodes. With repeated real-time measurements before an actual lapse occurred, the authors were able to show that sudden stressors were better predictors of smoking lapses than longer-lasting background stress. In a similar manner, Ferguson and Shiffman [12•] showed that negative affect was increasing in the hours preceding a smoking lapse episode. Thus, as demonstrated in these studies, EMA can be a highly useful tool for understanding the temporal relationships that exist between variables of interest.

\section{Contextual Associations}

An individual's behavior is influenced by the interplay that occurs between environmental and contextual factors [13]. EMA is able to capture these interactions in a way that laboratory-based research cannot, because data are collected in the participant's natural environment. It is for this reason that EMA lends itself particularly well to the study of substance use and dependence: Events such as relapse can be assessed with a degree of accuracy and a depth of detail that was previously unattainable. Moreover, by repeatedly sampling variables of interest within individuals and over time, EMA can be used to demonstrate the moment-by-moment changes that occur in an individual's behavior in response to contextual factors. Shiffman and colleagues [14], for instance, observed that exposure to environmental stimuli, such as alcohol or the presence of other smokers, as well as negative affect states, significantly increased the momentary risk of relapse during quit attempts. Likewise, Piasecki et al. [15] showed that alcohol use affects positive reinforcement ratings of subsequently smoked cigarettes. In a similar way, daily hassles were shown to be yet another important contextual factor predicting craving in smokers [16]. In this manner, EMA methods can be used to understand the role of context with a degree of detail that is otherwise unattainable.

\section{Within-Individual Change}

A fundamental premise underlying EMA research is that many behavioral processes are subject to within-individual variability and cannot be generalized from means derived at the population level $[2,17,18]$. To truly understand withinindividual change, the researcher needs assessment methods that obtain accurate moment-by-moment within-individual data, rather than seeking to make assessments based upon large samples of individuals. Because EMA methods repetitively sample variables of interest over time, multiple data points (often hundreds or more) are collected for each individual. Hedeker, Mermelstein, and Demirtas [19] demonstrated different research questions that can be addressed by looking at between- and/or within-subjects analyses of affect among smokers and non-smokers.

Differences in behavioral determinants (e.g., effect, attention) between individuals do not necessarily reflect variations within individuals. For example, Waters et al. [20] showed that attentional bias toward smoking cues was related to higher craving on the between-subjects level; however, this effect was not found on the within-subjects level: Instances where an individual's attentional bias was higher than the individual's average bias score were not related to higherthan-average cravings for the individual. This contradicts theoretical models which assume that higher attentional bias goes along with higher cravings. The authors suggest that this may have important implications for treatments: While treatments that reduce attentional bias may reduce craving in smokers who generally exhibit a high attentional bias, this might not be the case in episodes where the smoker exhibits a bias that is higher than usual. Such conclusions can only be reached when looking at within-subjects variations using multilevel modeling, and this is only possible with a potentially large amount of repeated measurements.

\section{Natural History}

EMA provides a useful means of obtaining longitudinal data. Long-term laboratory-based research is often impractical while, as previously discussed, retrospectively attained data is likely to be subject to bias and error. EMA is perfectly suited to longer-term data collection within a participant's natural environment. This is particularly useful when studying withdrawal symptoms over time [e.g., 21], effects and mechanisms of treatments [e.g., 22], or patterns of smoking over time [23•]. The latter study [23•], for example, used EMA methods to explore smoking patterns over the course of 3 weeks and, in particular, the role that environmental cues play in prompting smoking (stimulus control). Typically, stimulus control is explored in tightly controlled laboratory settings; but such studies suffer from limitations related to the artificial nature of laboratory settings (e.g., ecological validity).

Intervention

Recently, there has been an interest in using EMA not only to assess momentary experience, but also to influence change in the participant through generic motivational messages or, more recently, tailored interventions that respond to the individual's circumstances. For example, Wetter and colleagues 
[24] used palmtop computers to assess different dimensions of high-risk relapse situations and subsequently delivered individualized, context-specific coping strategies in addition to general quit smoking information and strategies as well as motivational messages. Thus, EMA is demonstrating its potential to become an active health intervention tool that combines assessment and treatment to respond to individual participants' contexts, activities, and moods [for a review, see 25].

In summary, EMA is an indispensible data collection tool when trying to answer research questions regarding temporal sequences, contextual associations, and how the individual changes over time. These questions would be difficult, if not impossible to study using other, more traditional forms of data collection.

\section{Assessment Methods and Considerations}

EMA aims to produce reliable data that is representative of participants' daily lives. Like any other research methodology, EMA protocols must be carefully designed to ensure they will address the research questions and hypotheses by gathering reliable data, in the right quantity and frequency, and without placing an undue burden upon research participants. This section provides examples of EMA technologies, with special attention to the electronic devices and software that have become prevalent in EMA studies. It then examines the growing versatility of EMA methods, and the huge variety of data that are now possible to collect in this manner. Finally, it discusses issues that researchers need to take into consideration when designing EMA protocols.

\section{Novel Technologies and Assessment Methods}

Intensive daily assessment can be done via a diverse range of technologies; research has been conducted via ambulatory physiological monitoring [26], palmtop computers [14], telephone calls or "hotlines" [27], and even instrumented pill bottles [28], to name just a few. Electronic methods have largely replaced paper-based diaries; primarily because it is difficult to assess whether participants have completed them in real time or retrospectively. Indeed, a landmark study by Stone et al. [29], called into doubt the reliability of paperbased diaries by using a paper-based diary that, without the participants' knowledge, date- and time-stamped their entries. The results showed that despite a $90 \%$ reported compliance with the protocol, actual compliance was only $11 \%$. In contrast, a parallel group using electronic diaries achieved $94 \%$ actual compliance. The authors concluded that paper diary participants had faked compliance by "hoarding" and completing their diary entries retrospectively, rather than at the required time. In contrast, electronic diaries, that record when (and sometimes where) diary entries are completed, are far more reliable. Furthermore, electronic diaries are also a boon to the researcher because the data is created in an electronic format, without the need to transcribe participants' diary records.

The evolution of handheld technologies, and in particular, the near-global availability of smartphones, has contributed to the growing popularity and utility of EMA methods. EMA studies conducted using smartphones are generally equipped with study-specific software, or study-specific apps, and a range of off-the-shelf packages are also available [see 30]. An advantage of using apps that participants can download onto their own devices is that the study does not need to meet the hardware and logistical costs of supplying participants with devices that they must remember to carry (and keep charged) in addition to their own. The costs incurred in providing study-specific devices can be significant, and in our experience about up to $20 \%$ will be lost or damaged [13]. A potential disadvantage of relying on participants' own devices is that not all participants have appropriate devices, potentially limiting participants or biasing samples, for example, by excluding older or poorer people. Participants may also be distracted by calls to their phone or other phone applications and forget to record incidents or complete surveys. Finally, compatibility issues with different operating platforms and privacy concerns about downloading information from participants' devices can mean that supplying study-specific devices is more cost effective.

Another change associated with the increasing availability of handheld technologies that is particularly advantageous to the researcher, is in the variety of data that is now possible to collect. Participants can use smartphones to take photographs, record videos, or make audio recordings, in addition to traditional self-report information such as diaries, surveys, and event logs. Dunton and colleagues [31] have combined EMA with objective assessments of physical activity making use of the built-in motion sensor in smart phones - a data collection strategy they call context-sensitive momentary assessment (CS-EMA). This is superior to previous research about people's day-to-day physical activity which has relied upon retrospective recall and/or activity monitors, which are not equipped to gather behavioral and contextual details, such as the type of sedentary or physical activity that was being performed, or to link the activities with mood, behaviors, and context. Thus, CS-EMA makes use of other mobile technologies to attain an even greater variety of data from participants' day-to-day lives. Apart from physical activity, other physiological responses, such as blood-glucose readings from diabetic patients, heart rate, or sleep may feasibly be recorded.

In a similar manner, EMA methods may be used concurrently with global positioning systems (GPS) to gather geolocation data. This enables researchers to obtain accurate 
information about individual participant's movements, which, when combined with diary and survey information, provides a rich dataset to understand place-based questions. Kirchner et al. [32•], for instance, used GPS technology to record the geographical movements of participants who were attempting to quit smoking. The GPS records allowed the authors to determine when and how often participants encountered places where tobacco was sold. This data was compared with self-reported craving levels and smoking status to assess whether there was a relationship between the number of times tobacco outlets were encountered and smoking lapses.

These examples demonstrate the enormous potential of EMA methodologies for the study of smoking and other health-related behaviors. Study designs that combine EMA methods with other mobile technologies have the potential to provide insight into all manner of environmental or social phenomena and their influence on smoking behavior.

\section{Design Considerations}

While EMA will prove a suitable method to assess many phenomena, it has some limitations and is not suited to all research questions. The following section will review some of the more important considerations researchers must make when designing EMA protocols.

\section{Assessment protocols}

Assessments need to reflect an underlying theory of change; however, very often, little is known about how fast or slowly changes in one variable (e.g., social cognitions) may influence changes in health behaviors. Therefore, it is difficult to determine ideal points of measurement in the planning phase of an EMA study. For example, assessments that are too frequent may easily become a burden to participants while not necessarily delivering useful information to the researcher [33]. Another challenge may be using EMA to assess behaviors and phenomena that are infrequent or rare. Nevertheless, there are studies that successfully sampled infrequent events such as cigarette consumption in non-daily smokers [34]. In order to obtain enough power when assessing such infrequent events, longer study duration may be warranted. Additionally, high diligence is required in training participants to be able to identify and $\log$ all of these events so that none of them are missed. Another challenge of EMA research arises from the fact that due to the high frequency of assessments, each single assessment has to be rather brief. This raises concerns regarding the psychometric properties of the items used. To date, there is a dearth of research testing the reliability and validity of the commonly very short measures used in EMA research. Apart from these measurement issues, participants with limited literacy and/or information technology skills or poor motor coordination may also struggle to use EMA technologies, although emerging voice-activated applications may overcome these problems in the future. The nature of the research subject is also an important consideration. Some participants may find it difficult to comply with EMA, if for example they are typically disorganized, lead chaotic lives, are generally non-compliant, or do not follow instructions.

\section{Compliance}

An effective EMA design must balance the data requirements of researchers with the needs of the participants. Phenomena or target episodes will ideally be sampled prior to, during, and post episode. However, if the sampling density is too high, or the information that must be provided at each sample is too much, participants may become irritated and/or reluctant to comply with the EMA protocol [35]. Participant compliance may be a significant challenge for researchers, with the potential of rendering their data invalid [36, 37]. Failure to document events or complete EMA surveys may also arise from forgetfulness rather than resistance to the study, particularly in the case of target events that are frequent or not particularly important to the participant [38].

While compliance issues are not unique to EMA studies, care must be taken to design EMA protocols that enhance compliance through good design and by motivating participants to comply. Indeed, participant motivation and rapport is a crucial element. Researchers should take into account the resources required to maintain relationships and encourage compliance throughout the planned study. For instance, Crayen et al. [39] provided participants with varying amounts of money dependent on their performance. The effects of noncompliance upon the validity of the data will depend on several factors, including the degree of non-compliance and whether non-compliance is biased in a particular direction. For example, if smokers tend to underreport cigarettes smoked when they were in a bad mood, then data collected would not accurately represent the relationship between smoking and mood and subsequent conclusions might not be valid.

Another challenge for EMA research is the increased costs of data management, analysis, and technology over traditional assessment methods. Technological developments such as the decreasing cost of smartphones and the use of EMA apps that can be downloaded onto participants' own phones will render EMA research more cost effective in the future. Information is recorded electronically, avoiding data transcription and conversion costs. But one of the possible disadvantages of EMA is that such studies produce a huge amount of data that may prove difficult to manage and process to a stage where it can be analyzed. Detecting errors, cleaning and standardizing data, and preparing it for transfer to statistical packages may prove more complex and time intensive than expected. Moreover, appropriate statistical methods to analyze such 
data require a good statistical understanding [although see 40 for an excellent overview on different modeling techniques].

\section{Conclusions}

Ecological momentary assessment enables researchers to obtain real-time information about individuals in their lived environment. EMA has provided researchers with tools that enable them to observe participants' lives as they are lived, rather than in laboratory settings. Using EMA, participants can record information about events, situations, thoughts, feelings, and behaviors as they occur. Both in isolation and in concert with other technologies such as geospatial software, symptom monitors, and video, researchers can use EMA methods to gather rich data about individuals' daily lives. Self-reported data provided via EMA overcomes some of the recall problems attendant with data that is recorded retrospectively. It also enables participants to record events as they unfold over time, providing researchers with the opportunity to obtain a high degree of temporal resolution about the natural history of the phenomena under investigation. As such, we can learn more about the dynamic relationships between individuals and their changing contexts and environments.

EMA has proven to be a highly versatile research methodology and is applicable to the study of many health behaviors. It has shown particular utility in the area of dependence and addiction research. However, despite its many strengths, EMA is not an appropriate tool for all research topics. The key criterion for deciding to use EMA methods is whether or not the target phenomenon is rarely occurring or particularly stable over time; EMA is not appropriate for the study of phenomena that occur infrequently or show little variability. EMA methods may also present challenges to participants who have poor literacy or information technology skills, or who are unable to follow instructions, although EMA has been used successfully with such populations.

Despite these limitations, there is still a substantial scope to apply EMA to the study of smoking behaviors. Their application will continue to shed light on the processes that underpin smoking-relevant experiences and behaviors; insights that may lead to improvements in interventions aiming at shaping and encouraging smoking cessation and preventing uptake. New methods that take advantage of developments in portable information technologies suggest that EMA will broaden its applicability and usefulness to study people's behaviors and experiences and improve our understanding of smoking behavior.

Acknowledgments The authors are grateful to Isabelle Morris for her editorial assistance.

\section{Compliance with Ethics Guidelines}

Conflict of Interest Natalie Schüz, John Cianchi, and Stuart G Ferguson declare that they have no conflict of interest.

Saul Shiffman consults to and has an interest in eRT, Inc., which provides electronic diaries for research.

Human and Animal Rights and Informed Consent This article does not contain any studies with human or animal subjects performed by any of the authors.

\section{References}

Papers of particular interest, published recently, have been highlighted as:

- Of importance

1. Stone AA, Shiffman S. Ecological momentary assessment (EMA) in behavioral medicine. Ann Behav Med. 1994;16(3):199-202.

2. Shiffman S, Stone AA, Hufford MR. Ecological momentary assessment. Annu Rev Clin Psychol. 2008;4:1-32.

3. Stone AA, Shiffman S, Atienza AA, Nebeling L. Historical roots and rationale of ecological momentary assessment (EMA). The science of real-time data capture: self-reports in health research. New York: Oxford University Press; 2007. p. 3-10.

4. Reis HT, Gosling SD. Social psychological methods outside the laboratory. Handbook of social psychology. 2010.

5. Berkman ET, Falk EB, Lieberman MD, 4. In the trenches of realworld self-control: neural correlates of breaking the link between craving and smoking. Psychol Sci. 2011;22(4):498-506. First study to integrate EMA with neuroimaging methods in a self-regulation context.

6. Schwarz N. Retrospective and concurrent self-reports: The rationale for real-time data capture. The science of real-time data capture: self-reports in health research. 2007:11-26

7. Conner TS, Barrett LF. Trends in ambulatory self-report: the role of momentary experience in psychosomatic medicine. Psychosom Med. 2012;74(4):327-37.

8. Redelmeier DA, Kahneman D. Patients' memories of painful medical treatments: real-time and retrospective evaluations of two minimally invasive procedures. Pain. 1996;66(1):3-8.

9. Tversky A, Kahneman D. Judgment under uncertainty: heuristics and biases. In: Tversky A, Kahneman D, editors. Judgment under uncertainty: heuristics and biases. Cambridge: Cambridge University Press; 1982. p. 3-20.

10. Shiffman S. Conceptualizing analyses of ecological momentary assessment data. Nicotine Tob Res. 2014;16 Suppl 2:S76-87.

11. Shiffman S, Waters AJ. Negative affect and smoking lapses: a prospective analysis. J Consult Clin Psychol. 2004;72(2):192-201.

12. Ferguson SG, Shiffman S. Effect of high-dose nicotine patch on craving and negative affect leading up to lapse episodes. Psychopharmacology. 2014, Advance online publication. Explores craving and negative affect levels in the hours before a lapse episode. First study to show that craving and affect trajectories before a lapse are similar in treated (using nicotine patches) and untreated (using placebo patches) quit attempts.

13. Ferguson SG, Shiffman S. Using the methods of ecological momentary assessment in substance dependence research — smoking cessation as a case study. Subst Use Misuse. 2011;46(1):87-95. 
14. Shiffman S, Paty JA, Gnys M, Kassel JA, Hickcox M. First lapses to smoking: within-subjects analysis of real-time reports. J Consult Clin Psychol. 1996;64(2):366-79.

15. Piasecki TM, Jahng S, Wood PK, Robertson BM, Epler AJ, Cronk NJ, et al. The subjective effects of alcohol-tobacco co-use: an ecological momentary assessment investigation. J Abnorm Psychol. 2011;120(3):557-71.

16. Volz AR, Dennis PA, Dennis MF, Calhoun PS, Wilson SM, Beckham JC. The role of daily hassles and distress tolerance in predicting cigarette craving during a quit attempt. Nicotine Tob Res. 2014;16(6):872-5.

17. Hamaker E. Why researchers should think "within-person": a paradigmatic rationale. In: Mehl M, Conner T, editors. Handbook of research methods for studying daily life. New York: The Guilford Press; 2012. p. 43-61.

18. Molenaar PCM. A manifesto on psychology as idiographic science: bringing the person back into scientific psychology, this time forever. Meas Interdiscip Res Perspect. 2004;2(4):201-18.

19. Hedeker D, Mermelstein RJ, Demirtas H. Modeling betweensubject and within-subject variances in ecological momentary assessment data using mixed-effects location scale models. Stat Med. 2012;31(27):3328-36.

20. Waters AJ, Szeto EH, Wetter DW, Cinciripini PM, Robinson JD, Li Y. Cognition and craving during smoking cessation: an ecological momentary assessment study. Nicotine Tob Res. 2014;16 Suppl 2: S111-8.

21. Shiffman S, Patten C, Gwaltney C, Paty J, Gnys M, Kassel J, et al. Natural history of nicotine withdrawal. Addiction. 2006;101(12): 1822-32.

22. Schüz N, Ferguson S. An exploratory examination of the mechanisms through which pre-quit patch use aids smoking cessation. Psychopharmacology. 2014:1-7

23. Shiffman S, Dunbar MS, Li XX, Scholl SM, Tindle HA, Anderson SJ, et al. Smoking patterns and stimulus control in intermittent and daily smokers. Plos One. 2014;9(3):14. Compares patterns and antecedents of smoking in intermittent smokers and daily smokers. Results suggest that intermittent smoking is under much stronger stimulus control than daily smoking.

24. Wetter DW, McClure JB, Cofta-Woerpel L, Costello TJ, Reitzel LR, Businelle MS, et al. A randomized clinical trial of a palmtop computer-delivered treatment for smoking relapse prevention among women. Psychol Addict Behav. 2011;25(2):365-71.

25. Kaplan RM, Stone AA. Bringing the laboratory and clinic to the community: mobile technologies for health promotion and disease prevention. Annu Rev Psychol. 2013;64(1):471-98.

26. Kop WJ, Verdino RJ, Gottdiener JS, O'Leary ST, Merz CNB, Krantz DS. Changes in heart rate and heart rate variability before ambulatory ischemic events. J Am Coll Cardiol. 2001;38(3):742-9.
27. Perrine MW, Mundt JC, Searles JS, Lester LS. Validation of daily self-reported alcohol consumption using interactive voice response (IVR) technology. J Stud Alcohol. 1995;56(5):487-90.

28. Byerly MJ, Fisher R, Carmody T, Rush AJ. A trial of compliance therapy in outpatients with schizophrenia or schizoaffective disorder. J Clin Psychiatry. 2005;66(8):997-1001.

29. Stone AA, Shiffman S, Schwartz JE, Broderick JE, Hufford MR. Patient compliance with paper and electronic diaries. Control Clin Trials. 2003;24(2):182-99.

30. Barta W, Tennen H, Litt M. Measurement reactivitiy in diary research. In: Mehl M, Conner T, editors. Handbook of research methods for studying daily life. New York: The Guilford Press; 2012. p. 108-23.

31. Dunton GF, Dzubur E, Kawabata K, Yanez B, Bo B, Intille S. Development of a smartphone application to measure physical activity using sensor-assisted self-report. Frontiers in Public Health. 2014;2

32. Kirchner TR, Cantrell J, Anesetti-Rothermel A, Ganz O, Vallone DM, Abrams DB. Geospatial exposure to point-of-sale tobacco: real-time craving and smoking cessation outcomes. Am J Prev Med. 2013;45(4):379-85. First application of EMA using GPS to study the interaction craving and exposure to point-of-sale tobacco.

33. Collins LM. Analysis of longitudinal data: the integration of theoretical model, temporal design, and statistical model. Annu Rev Psychol. 2006;57(1):505-28.

34. Shiffman S, Ferguson SG, Dunbar MS, Scholl SM. Tobacco dependence among intermittent smokers. Nicotine Tob Res. 2012;14(11): 1372-81.

35. Hacker ED, Ferrans CE. Ecological momentary assessment of fatigue in patients receiving intensive cancer therapy. J Pain Symptom Manage. 2007;33(3):267-75.

36. Courvoisier DS, Eid M, Lischetzke T. Compliance to a cell phonebased ecological momentary assessment study: the effect of time and personality characteristics. Psychol Assess. 2012;24(3):71320.

37. Schüz N, Walters JAE, Frandsen M, Bower J, Ferguson SG. Compliance with an EMA monitoring protocol and its relationship with participant and smoking characteristics. Nicotine Tob Res. 2014;16:S88-92.

38. Shiffman S. Designing protocols for ecological momentary assessment. In: Stone A, Shiffman S, Atienza AA, Nebeling L, editors. The science of real-time data capture: self-reports in health research. New York: Oxford University Press; 2007. p. 27-53.

39. Crayen C, Eid M, Lischetzke T, Courvoisier DS, Vermunt JK. Exploring dynamics in mood regulation - mixture latent Markov modeling of ambulatory assessment data. Psychosom Med. 2012;74(4):366-76.

40. Bolger N, Laurenceau J-P. Intensive longitudinal methods. New York: The Guilford Press; 2013. 